Pneumologe $2021 \cdot 18: 239-240$

https://doi.org/10.1007/s10405-021-00397-y

Angenommen: 12. Mai 2021

Online publiziert: 28. Mai 2021

(c) Springer Medizin Verlag $\mathrm{GmbH}$, ein Teil von Springer Nature 2021

\section{Redaktion}

Monika Gappa, Düsseldorf

Markus A. Rose, Stuttgart

Martin Rosewich, Oberursel
Markus A. Rose

Pädiatrische Pneumologie, Allergologie und CF-Zentrum, Klinikum Stuttgart, Standort Mitte

(Olgahospital), Stuttgart, Deutschland

\title{
Aktuelles Konsensuspapier zu „Induzierbare laryngeale Obstruktion (ILO)"
}

\section{Hinweis auf das Positionspaper der Arbeitsgruppe „Dysfunktionelle Respiratorische Symptome“ der Gesellschaft für Pädiatrische Pneumologie (GPP)}

Seit dem 19. Jahrhundert gibt es Berichte über funktionelle laryngeale Störungen mit dem Leitsymptom „Stridor“; 1974 erhob Patterson den ersten bronchoskopischen Befund einer paradoxen Stimmbandaktivität. Nachdem zwischenzeitlich in den 1970er-Jahren ein rein psychiatrisches Pathogenesekonzept i. S. einer „Hysterie“ nach Psychotraumata dominiert hatte, führte Christopher in den 1980er-Jahren die Entität „vocal cord dysfunction“ (VCD) ein. In 2017 empfahl die ERS den Oberbegriff ,inducible laryngeal obstruction (ILO)“, während in HNO-Ärztekreisen von „paradoxer Stimmbandbeweglichkeit“ („paradoxical vocal fold motion“ [PVFM]) die Rede ist. Diese häufigen, oft funktionellen inspiratorischen Stimmbandadduktionen gehen mit laryngealer Überempfindlichkeit bis $\mathrm{zu}$ Laryngospasmus und Obstruktion, Dysphonie und Husten einher. Klinisch imponieren ein oft inspiratorischer oder biphasischer Stridor, Sprechschwierigkeiten und Ängstlichkeit. Die Ätiologie ist eher funktionell als psychogen; somatische Faktoren können Entzündung, gastroösophagealer Reflux und Virusinfektionen sein. Eine in der symptomatischen Episode beweisende Laryngoskopie ist der diagnostische Goldstandard mit (ggf. unter Belastung auftretender) Stimmbandadduktion in
Inspiration bei ansonsten normalem Befund.

Die Differenzialdiagnose bei den bevorzugt weiblichen Betroffenen umfasst Asthma bronchiale, allergische Reaktionen, Stimmbandanomalien und KruppSyndrom; neben Fehldiagnosen gibt es auch Überlappungen, da bis zu $50 \%$ der Asthmatiker gleichzeitig eine PVFM aufweisen. Fehl- bzw. Überbehandlungen sind häufig und reichen von erfolgloser jahrelanger Inhalation bis zu unnötigen endotrachealen Intubationen oder chirurgischen Interventionen.

Bei rein funktionellem Charakter fokussiert die Behandlung auf Schulung der Betroffenen über die relative Harmlosigkeit der Beschwerden und dem (Wieder-)Erlernen die Stimmbänder entspannender normaler Atemtechniken, optional unterstützt durch visualisierte Biofeedbackmethoden. Schon in den 1950er-Jahren hatte Dr. Buteyko Atemtechniken für Asthmatiker beschrieben, die objektivierbar zu besserer Symptomkontrolle führten. Diese auf nasaler Atmung, Atemanhalten und Entspannungsübungen beruhenden Techniken konnten später von Blager, Gay und Wood (1988) erfolgreich bei habituellem Husten und „vocal cord dysfunction“ eingesetzt werden. Bei sehr schweren Formen können Pharmaka und laryn- 
geale Botulinumtoxin-Injektionen zur Anwendung kommen.

Das Positionspapier der GPP-Arbeitsgruppe „Dysfunktionelle respiratorische Symptome“ gibt einen schönen Überblick zum aktuellen Kenntnisstand und $\mathrm{zu}$ Vorgehensweisen bei diesem in der pneumologischen Praxis wichtigen faszinierenden Krankheitsbild. Es ist in der Monatsschrift Kinderheilkunde erschienen und ist unter https://doi. org/10.1007/s00112-021-01159-z und www.springermedizin.de/link/10.1007/ s00112-021-01159-z für alle Interessierten frei zugänglich [1].

\section{Korrespondenzadresse}

\section{Prof. Dr. med. Dr. med. habil.}

Markus A. Rose, M.P.H.

Pädiatrische Pneumologie, Allergologie und

CF-Zentrum, Klinikum Stuttgart, Standort Mitte (Olgahospital)

Kriegsbergstr. 62, 70174 Stuttgart, Deutschland m.rose@klinikum-stuttgart.de

\section{Einhaltung ethischer Richtlinien}

Interessenkonflikt. M.A. Rose gibt an, dass kein Interessenkonflikt besteht.

Für diesen Beitrag wurden vom Autor keine Studien an Menschen oder Tieren durchgeführt. Für die aufgeführten Studien gelten die jeweils dort angegebenen ethischen Richtlinien.

\section{Literatur}

1. Dillenhöfer S, Hinrichs B, Kohl A et al (2021) Die induzierbare laryngeale Obstruktion (ILO) Ursachen, klinische Präsentation, Diagnostik und Therapie. Monatsschr Kinderheilkd. https://doi. org/10.1007/s00112-021-01159-z

\section{Fachnachrichten}

SPENDENLAUF 2021 für herzkranke Kinder www.ichlaufumdeinleben.de
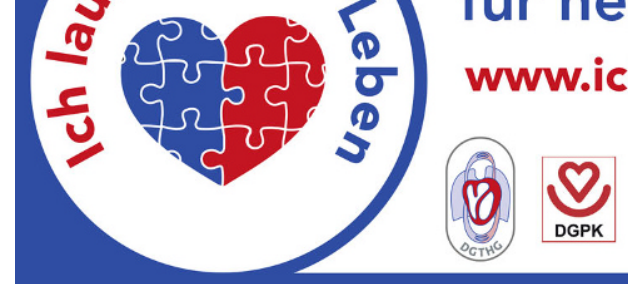

\section{$A B$ 11.01.2021}

\section{Ich lauf um Dein Leben - Spendenlauf für Kinderherz- transplantationen}

Mit der Aktion „Ich lauf um Dein Leben" rufen die Deutsche Gesellschaft für Thorax-, Herz- und Gefäßchirurgie e. V. (DGTHG) und die Deutsche Gesellschaft für Pädiatrische Kardiologie und Angeborene Herzfehler e. V. (DGPK) zu einem virtuellen Spendenlauf auf. Mit dem Spendenlauf sollen Kinder unterstützt werden, die dringend eine Herztransplantation brauchen.

Schwerst herzkranke Kinder warten häufig viele Monate bzw. mehr als ein Jahr auf ein passendes Spenderherz. Aktuell sind es 50 Mädchen und Jungen bundesweit. Andererseits werden weniger als 30 Spenderherzen jährlich in Deutschland transplantiert. Die Herausforderungen sowie Ängste und Sorgen, mit denen die kleinen Patientinnen und Patienten mit ihren Familien leben müssen, sind immens.

Um auf dieses wichtige Thema besonders aufmerksam zu machen, haben die Deutsche Gesellschaft für Thorax-, Herzund Gefäßchirurgie e.V. (DGTHG) und die Deutsche Gesellschaft für Pädiatrische Kardiologie und Angeborene Herzfehler e.V. (DGPK) daher am 11. Januar 2021 einen virtuellen Spendenlauf gestartet. Damit wollen die herzmedizinischen Fachgesellschaften im Rahmen ihrer digitalen Jahrestagungen Herzmedizin 2021 speziell das Thema Kinderherztransplantationen ins Bewusstsein der Öffentlichkeit rufen, um durch die Spenden Kindern, die ein Spenderorgan benötigen, noch gezielter helfen zu können. Der Erlös wird das bundesweit tätige Aktionsbündnis Angeborene Herzfehler e.V. unterstützen, in dem sechs Patientenvertretungen für angeborene Herzfehler vereint sind, um mit gemeinsamen Kräften Kindern, Jugendlichen und Erwachsenen mit angeborenen Herzfehlern zu helfen.

Mitmachen kann jeder: Der Erwerb des "Ich-lauf-um-dein-LebenLaufshirts" (https://www.tshirt-drucker.de/c/spendenlauf) impliziert bereits eine Spende und stattet die Läufer*innen passend für diesen Anlass aus; ebenfalls möglich und willkommen ist auch eine direkte Spende.

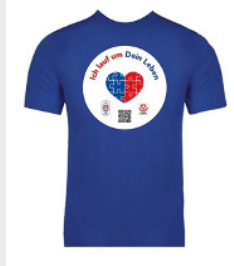

Der finale Jahreserlös der Charity-Aktion wird zum 31.12.2021 vorgestellt.

Alle Informationen erhalten Sie unter: https://www.ichlaufumdeinleben.de

\section{Spendenkonto Kinderherztrans- plantationen (DGTHG):}

Kontoinhaber:

Deutsche Gesellschaft für Thorax-, Herz- und Gefäßchirurgie e.V. (DGTHG)

Pax-Bank eG

IBAN: DE64 370601936005727004

BIC: GENODED1PAX

Stichwort: „Ich lauf um Dein Leben 2021“

Weitere Informationen unter: https://www.dgthg-jahrestagung.de http://www.kinderkardiologie.org/ jahrestagung/ 